\title{
A CONJECTURE OF J. NAGATA ON DIMENSION AND METRIZATION ${ }^{1}$
}

\author{
BY PHILLIP A. OSTRAND
}

Communicated by R. P. Boas, March 10, 1965

Theorem 1. A metrizable space $X$ is of dimension $\leqq n$ if and only if $X$ admits a metric compatible with the topology which satisfies the condition $(n)$ : For any $n+3$ points $x, y_{1}, \cdots, y_{n+2}$ of $X$ there exist distinct indices $i, j$ such that $d\left(y_{i}, y_{j}\right) \leqq d\left(x, y_{j}\right)$.

In this paper we outline briefly a proof of Theorem 1, which was conjectured by J. Nagata [1].

By dimension we shall always mean covering dimension. A family of subsets of $X$ is discrete if each point of $X$ has a neighborhood which meets at most one member of the family. For a subset $A$ of $X$ and a family $\mathfrak{C}$ of subsets of $X$, let $S(A, \mathfrak{e})$ denote the union of $A$ and all those $C \in \mathbb{C}$ such that $C \cap A \neq \varnothing$. For each integer $n \geqq 0$, let

$$
\begin{aligned}
S^{n}(A, \mathfrak{C}) & = \begin{cases}A & \text { if } n=0 \\
S\left(S^{n-1}(A, \mathcal{C}), \mathcal{C}\right) & \text { if } n>0\end{cases} \\
{[\mathcal{C}]^{n} } & =\left\{S^{n}(C, \mathcal{C}): C \in \mathcal{C}\right\} .
\end{aligned}
$$

Let $X$ be a metrizable space of dimension $\leqq n$. For each positive integer $j$ there exist $n+1$ discrete families of open sets, $\mathfrak{u}_{j}^{1}, \mathfrak{u}_{j}^{2}, \cdots, \mathfrak{u}_{j}^{n+1}$ such that if $\mathfrak{u}_{j}=\bigcup_{i=1}^{n+1} \mathfrak{u}_{j}^{i}$, then:

(1) each $u_{j}$ covers $X$;

(2) for each $x \in X,\left\{S\left(x, \mathfrak{u}_{j}\right): j=1,2, \cdots\right\}$ is a neighborhood base at $x$;

(3) $\left[\mathfrak{u}_{j+1}\right]^{31}$ refines $u_{j}$ for each $j$;

(4) if $j<k$ and $1 \leqq i \leqq n+1$, each member of $\left[\mathfrak{u}_{k}\right]^{31}$ meets at most one member of $u_{j}^{i}$.

The $\mathfrak{u}_{j}^{i}$ are defined inductively on $j$. Their construction relies on a new characterization of dimension [2].

THEOREM 2. A metrizable space $X$ is of dimension $\leqq n$ if and only if for each open cover $\mathfrak{C}$ of $X$ there exist $n+1$ discrete families of open sets, $\mathfrak{u}^{1}, \mathfrak{u}^{2}, \cdots, \mathfrak{u}^{n+1}$ such that $\bigcup_{i=1}^{n+1} \mathfrak{u}^{i}$ is a cover of $X$ which refines $\mathfrak{C}$.

Proof of Theorem 1. Let $R^{*}$ denote the set of dyadic rationals in the open interval $(0,1)$. For each $m \in R^{*}$ there exist $n+1$ discrete ship.

1 This research was supported in part by a National Science Foundation Fellow- 
families of open sets $\delta_{m}^{1}, \delta_{m}^{2}, \cdots, \delta_{m}^{n+1}$ such that if $S_{m}=\bigcup_{i=1}^{n+1} S_{m}^{i}$ then:

$\left(1^{\prime}\right)$ each $S_{m}$ covers $X$

(2') for each $x \in X,\left\{S\left(x, S_{m}\right): m \in R^{*}\right\}$ is a neighborhood base at $x$;

$\left(3^{\prime}\right)$ if $m<p \in R^{*}$, then $S_{m}$ refines $S_{p}$;

$\left(4^{\prime}\right)$ if $m, p \in R^{*}$, and $j$ is a positive integer such that $2^{-j} \leqq m<p$ $\leqq 2^{-(j-1)}$, then $S_{m}^{i}$ refines $\delta_{p}^{i}$ for each $1 \leqq i \leqq n+1$;

$\left(5^{\prime}\right)$ if $m<p \in R^{*}, 1 \leqq i \leqq n+1$ and $U \in S_{m}^{i}, V \in s_{p}^{i}$, then either $U \subset V$ or $U \cap V=\varnothing$;

$\left(6^{\prime}\right)$ if $m, p \in R^{*}, m+p<1$, and if $U \in S_{m}, V \in S_{p}$ are such that $U \cap V \neq \varnothing$, then there exists $W \in S_{m+p}$ such that $U \cup V \subset W$.

The $S_{m}^{i}$ are constructed from the $\mathfrak{u}_{j}^{i}$ as follows: Let

$$
* \mathfrak{u}_{j}^{i}=\left\{S^{31}\left(U, \mathfrak{u}_{j+1}\right): U \in \mathfrak{u}_{j}^{i}\right\} ; \quad{ }^{*} \mathfrak{u}_{j}=\bigcup_{i=1}^{n+1} * \mathfrak{u}_{j}^{i} .
$$

For $A \subset X, 1 \leqq i \leqq n+1, j \geqq 1$ and $k \geqq 0$, let

$$
\begin{aligned}
& T^{k}(A, i, j)= \begin{cases}S\left(A,{ }^{*} \mathfrak{u}_{j}^{i}\right) & \text { if } k=0, \\
S\left(T^{k-1}(A, i, j),{ }^{*} \mathfrak{u}_{j+k}^{i}\right) & \text { if } k>0 ;\end{cases} \\
& T(A, i, j)=\bigcup_{k=0}^{\infty} T^{k}(A, i, j) .
\end{aligned}
$$

For $m \in R^{*}$ of the form $m=\sum_{k=1}^{t} 2^{-m_{k}}$, where $1 \leqq m_{1}<m_{2}<\cdots<m_{t}$, let $\bar{m}=\sum_{k=1}^{t-1} 2^{-m_{k}}$. Further, for $A \subset X$ and $1 \leqq i \leqq n+1$, let

$$
\begin{aligned}
i_{A} m & = \begin{cases}T\left(A, i, m_{1}+1\right) & \text { if } t=1, \\
T\left(S^{3}\left(i_{A} \bar{m},{ }^{*} \mathcal{u}_{m_{t}}\right), i, m_{t}\right) & \text { if } t>1 ;\end{cases} \\
S_{m}^{i} & =\left\{i_{U} m: U \in \mathcal{u}_{m_{1}}^{i}\right\} .
\end{aligned}
$$

This complicated construction is necessary to achieve condition $\left(5^{\prime}\right)$. A simpler construction in which $\left(5^{\prime}\right)$ does not hold was used by Nagata [1] to obtain a weaker result.

Define a non-negative real-valued function $d$ on $X \times X$ by

$d(x, y)= \begin{cases}1 & \text { if } y \notin S\left(x, \varsigma_{m}\right) \text { for any } m \in R^{*}, \\ \inf \left\{m \in R^{*}: y \in S\left(x, \varsigma_{m}\right)\right\} & \text { if } y \in S\left(x, \varsigma_{m}\right) \text { for some } m \in R^{*} .\end{cases}$

$d$ is clearly symmetric. It follows from $\left(3^{\prime}\right)$ that $d(x, y)=0$ only if $x=y$. The triangular inequality follows from $\left(6^{\prime}\right)$. Thus $d$ is a metric on $X$. By $\left(1^{\prime}\right)$ and $\left(2^{\prime}\right) d$ is compatible with the topology of $X$. If $x, y_{1}, \cdots, y_{n+2}$ are points of $X$, then by $\left(4^{\prime}\right)$ and $\left(5^{\prime}\right)$ there exist indices $i, j, i \neq j$, such that $d\left(y_{i}, y_{j}\right) \leqq d\left(x, y_{j}\right)$.

Conversely, suppose that $X$ is a metric space with metric $d$ satisfy- 
ing condition ( $\mathrm{n})$. Let $\mathfrak{e}$ be an open cover of $X$. It is easily shown by use of Zorn's Lemma that if $A \subset X$ and $\epsilon>0$, there is a subset $B$ of $X$ which is maximal (under inclusion) with respect to the properties:

(i) $B \cap A=\varnothing$;

(ii) $\left\{S_{\epsilon}(x): x \in B\right\}$ refines $\mathbb{e}:\left(S_{\epsilon}(x)=\{y \in X: d(x, y)<\epsilon\}\right)$

(iii) if $x \neq y \in B$, then $d(x, y) \geqq \epsilon$.

Hence we may construct subsets $A_{;}$of $X$ for $i=1,2, \cdots$, inductively on $i$, which are maximal with respect to the properties:

(1) $A_{i} \cap\left\{y \in X\right.$ : for some $1 \leqq j<i$ and $\left.x \in A_{j}, d(x, y)<2^{-j}\right\}=\varnothing$;

(2) $\left\{S_{2-i}(x): x \in A_{i}\right\}$ refines $\mathfrak{e}$;

(3) if $x \neq y \in A_{i}$, then $d(x, y) \geqq 2^{-i}$.

Let $\mathcal{u}=\left\{S_{2-i}(x): x \in A_{i} ; i=1,2, \cdots\right\}$. The maximality of the $A_{i}$ insures that $\mathcal{u}$ covers $X$. Conditions (1) and (3) and the condition on the metric insure that $u$ is of order $\leqq n+1$. Thus the proof is complete.

\section{REFERENCES}

1. J. Nagata, General topology and its relation to modern analysis and algebra, Academic Press, New York; pp. 282-285.

2. P. A. Ostrand, Dimension of metric spaces and Hilbert's Problem 13, Bull. Amer. Math. Soc. 71 (1964), 619-622.

NORTHWESTERN UNIVERSITY 